\title{
Quantitative Gait Analysis of Healthy Adults Using Foot Print Method and Win Track
}

\author{
Sumandeep Kaur ${ }^{1}$, VijJaspreet Singh $^{2}$ \\ ${ }^{1}$ MPT Student, Department of Physiotherapy, Lovely Professional University, India \\ ${ }^{2}$ Associate Professor, University College of Physiotherapy, Baba Farid University of Health Sciences, India
}

\begin{abstract}
Background: Analysis of gait is an integral part of study of human locomotion. It is an important aspect of health diagnostics, management and rehabilitation of numerous disorders. Various instruments have been developed for the analysis of gait parameters in due course of time. But to test its validity and reliability of is prior importance. Thus the purpose of the present study is to compare the foot print method of gait analysis with Win track platform.
\end{abstract}

Objective: To compare the various quantitative gait parameters using footprint method of gait analysis and Win track platform in healthy adults during bare foot walking.

Design: Experimental repeated measures, Convenient sampling.

Study Setting: Outpatients department, SBRMH, Lovely Professional University, Phagwara, India

Participants: Total 30 healthy participants(14 men and 16 women) were recruited for the study.

Procedure: All the subjects wereinstructed first to walk along a smooth horizontal $10 \mathrm{~m}$ long walkway. Only the middle five steps were calculated to avoid variability in the steps associated with initiation and termination of the gait. The gait velocity, cadence, step lengths and stride length were calculated for the quantitative gait analysis of the gait parameters. After that, the same subjects were instructed to walk on the WIN TRACK Platform. Three trials were taken for both groups and their averages were obtained.

Statistical Tool: SPSS VERSION 16

Result: There was a significant difference in the cadence ( $p$ value-0.000), gait velocity $(p$ value -0.001$)$ and stride length ( $p$ value -0.024). But no significant difference was observed in right step length ( $p$ value 0.205) and leftstep length(p value 0.128 ).

Conclusion: Although WIN TRACK has given almost similar values in terms of step lengths in comparison with foot print analysis, but it was unable to provide similar results in cadence, gait velocity and stride length, which are the integral part of human locomotion.

Keywords: Analysis of gait, Foot prints method of gait analysis, Gait parameters, WIN TRACK

\section{Introduction}

Gait analysis is an important aspect of human locomotion. Analysis of gait employs to measurement, description and assessment of quantities that characterize human locomotion or gait.[1] Gait analysis helps in the determination of kinetic and kinematic parameters of gait which in turn helps in the quantative analysis of musculoskeletal events occurring during locomotion. Thus gait analysis is an important aspect health diagnostics in the fields of rehabilitation, sports and health care. In sports the analysis of gait helps in improving the faulty athletic performance during the play. [2-6] Similarly in orthopedics gait analysis is done to know the biomechanical deviations during locomotion and also to know the healing progress. It helps in identifying the biomechanical deviations and can act as an important tool for assessment and treatment of musculoskeletal problems in orthopedic rehabilitation. It is an important tool of health medicine too. Gait analysis serve as an important indicator of neurological disease diagnosis, as of the neurological disorders represents a specific gait pattern. Thus gait analysis can act as a precursor for diagnosis. Also it can give the valuable information about the treatment of the disease and thus helps in improving the locomotion of patients. [7-12]

Numerous researches have been done on gait analysis. Gait analysis has become a widespread application in biomedical engineering and has become an area of keen interest among biomedical engineers. [13-17]A standard gait analysis method available is quiet expensive. It makes the use of multi camera motion capture with a force platform which is capable of recording ground reaction force. Although this type of analysis system is installed in many laboratories but it is expensive and limited to research zone only.[18-19]There is one another option available for gait analysis which uses wearable sensors in the shoes or waist of the patient, it is inexpensive and a popular mode of gait analysis. These sensors may be accelerometers, gyrosensors, force sensors, strain gauges, inclinometers, goniometers and soon. [20-21]

Human locomotion is a periodic activity of the body segments which involve the repetitive motions. Normal walking pattern is divided into eight different phases. These phases are: initial contact, loading 
response, midstance, terminal stance, pre-swing, initial swing, mid-swing, and terminal swing. Analysis of gait is of function significance because it will tell the motion generated at the different joints and body segments which in turns helps in the assessment, diagnosis and treatment of the particular condition. For the universal acceptance of gait analysis the Rancho Los Amigos gait analysis committee developed a generic terminology for the gait analysis in different phases. [22-26]

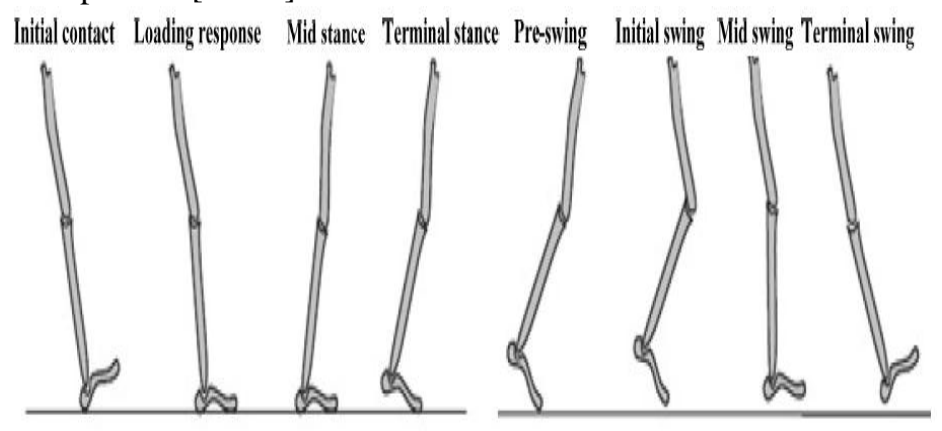

(a)

(b)

Figure 1: Gait cycle (a) stance period (b) swing period

Win track is a new instrument used for the measurement of planter pressures as well as gait parameters in barefoot walking. This instrument has been used in the research implications but still its validation and standardization needs to be performed. The instrument measures the gait parameters but if the results are reliable or not is needed to be confirmed.

The instrument consists of following dimensions: $1610 \mathrm{~mm} \times 652 \mathrm{~mm} \times 30 \mathrm{~mm}$ (length/width/height). The thickness of the platform is $9 \mathrm{~mm}$, and it consists of 12288 sensors, which are of resistive type. The dimensions of the sensors are $7.8 \times 7.8 \mathrm{~mm} 2$, and the acquisition frequency of the apparatus is up to 200 images/s. The data obtained from the sensors is directly uploaded on the computer attached to the instrument and the parameters are also computed by the same and the results are displayed on the screen.

Foot prints method of gait analysis is a standard and conventional method for analyzing the spatial parameters of gait cycle. Patient is made to walk after applying blue ink to his feet. Only middle five steps are counted on a 10 meter walkway. And all the spatial gait parameters are calculated.

Analysis of gait is an important aspect of assessment, diagnosis and management of a locomotion disorder. Thus the tool used for the analysis of gait parameters should have validity and reliability. So the present need of the study is to compare the gait parameters using foot print method of gait analysis as a standard with WIN TRACK platform. The study provides an insight that if WIN TRACK can be used as an instrument of choice for the assessment of gait parameters in various conditions.

\section{Materials And Methodology:}

A total of 30 asymptomatic participants, 20-40 years of age were included in the study. The subjects were taken from Lovely professional university. Age, gender, height $(\mathrm{cm})$, weight $(\mathrm{kg})$ and BMI were determined for each participant at the baseline.There demographic data were collected through individual interview. The inclusion criteria for selection was: (a) Age group of 20-40 years of age; (b) Right leg dominance; (c) BMI ranging from 18.5-24.9; (d) Co-operative and complained in gait analysis; (e) No history of fall; (f) MMSE score above 24. The subjects were excluded if they were having (a) any active medical problem; (b) deformities of spine and lower limb, such as flatfoot, pescavus, genu varum; (c) any neurological disorder; (d) any major orthopedic diagnosis involving lumbar spine, pelvis and lower extremities; (e) using walking aids; and (f) acute medical illness. All the subjects were assessed using MMSE. The study was explained to participants in detail and they were asked to sign a written consent form.

The participants were instructed to walk along a smooth horizontal $10 \mathrm{~m}$ long walkway with lateral borders at a comfortable speed. Only the middle five steps were evaluated to avoid the variable steps associated with initiation and termination of gait. The gait velocity, step length, stride length, cadence were measured for quantitative gait analysis. The step length $(\mathrm{cm})$ was measured from the geometrical heel centre of the current footprint to the same of the previous footprint on the opposite foot and stride length $(\mathrm{cm})$ from the line of progression between the heel points of two consecutive footprints of the same foot. The walking velocity $(\mathrm{cm} / \mathrm{s})$ was obtained after dividing the recorded distance by the ambulation time. The cadence was calculated by asking the subject to walk for one minute through a straight pathway with self selected speed, then calculating the no. of steps taken during 1 minute. This distance was independent of $10 \mathrm{~m}$ walkway. Each participant was given one prior walking before final assessment to become familiarized with walking condition. Footprint method was 
used for measuring the spatial gait parameters. Participants were asked to walk after applying blue ink on their feet to get the foot prints demonstrated in figure 2 .

Then after the footprint method of gait assessment the same participants were asked to walk on the WIN TRACK system, medicaptures, France. It consists of a platform ofdimensions $1610 \mathrm{~mm} \times 652 \mathrm{~mm} \times 30$ $\mathrm{mm}$ (length/width/height). Each participant was given one prior walking before final assessment to become familiarized with walking condition as in figure 3 .

For both the methods three trials were taken for all the parameters and there averages were obtained. The whole procedure occurred during a single session assessment that last for approximately 30 minutes for each participant.

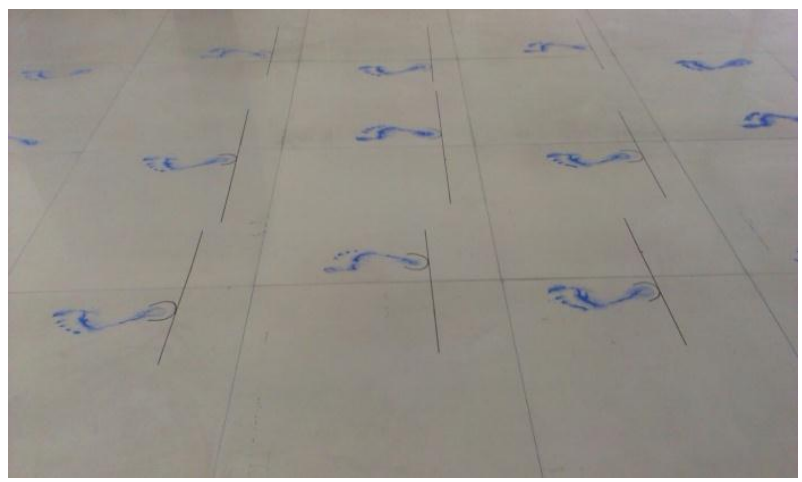

Figure 2: Foot prints method of gait analysis.

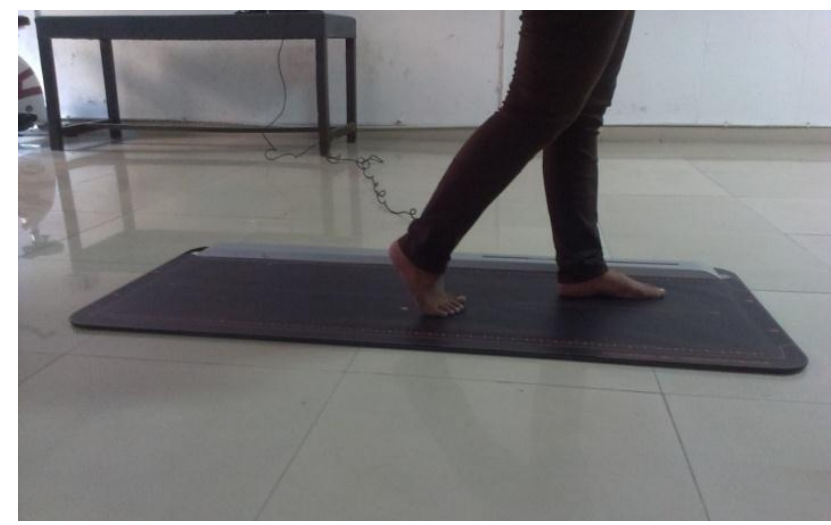

Figure 3: Participant walking on WIN TRACK platform

\section{Results:}

The result of the study showed that there was a significant difference in the gait velocity, cadence and stride length of the same participants when the parameters were analysed using foot prints method of gait analysis and WIN TRACK platform.

Table 1: Comparison of gait parameters using footprints method of gait analysis and WIN TRACK platform:

\begin{tabular}{|c|c|c|c|c|}
\hline Variables & $\begin{array}{c}\text { Mean } \pm \text { SD } \\
\text { (Foot prints analysis) }\end{array}$ & $\begin{array}{c}\text { Mean } \pm \text { SD } \\
\text { (WIN TRACK } \\
\text { analysis) }\end{array}$ & t value & p value \\
\hline Right Step length $(\mathrm{cm})$ & $52.2 \pm 16.7$ & $52.1 \pm 11.5$ & 1.296 & $0.205(\mathrm{NS})$ \\
\hline Left Step length $(\mathrm{cm})$ & $53.5 \pm 7.1$ & $51.8 \pm 6.1$ & 1.57 & $0.128(\mathrm{NS})$ \\
\hline Stride length $(\mathrm{cm})$ & $107.2 \pm 15.2$ & $100.5 \pm 12.6$ & 2.39 & $.024(\mathrm{~S})$ \\
\hline Cadence $($ Steps/min) & $101.2 \pm 10.2$ & $220.1 \pm 55.7$ & -4.17 & $.000(\mathrm{~S})$ \\
\hline Gait velocity $(\mathrm{cm} / \mathrm{s})$ & $90.8 \pm 16.1$ & $187.2 \pm 34.4$ & -3.89 & $.001(\mathrm{~S})$ \\
\hline
\end{tabular}

$\mathrm{S}=$ Significant

NS $=$ Non significant 
Figure 4: Comparison of gait parameters using footprints method of gait analysis and WIN TRACK platform

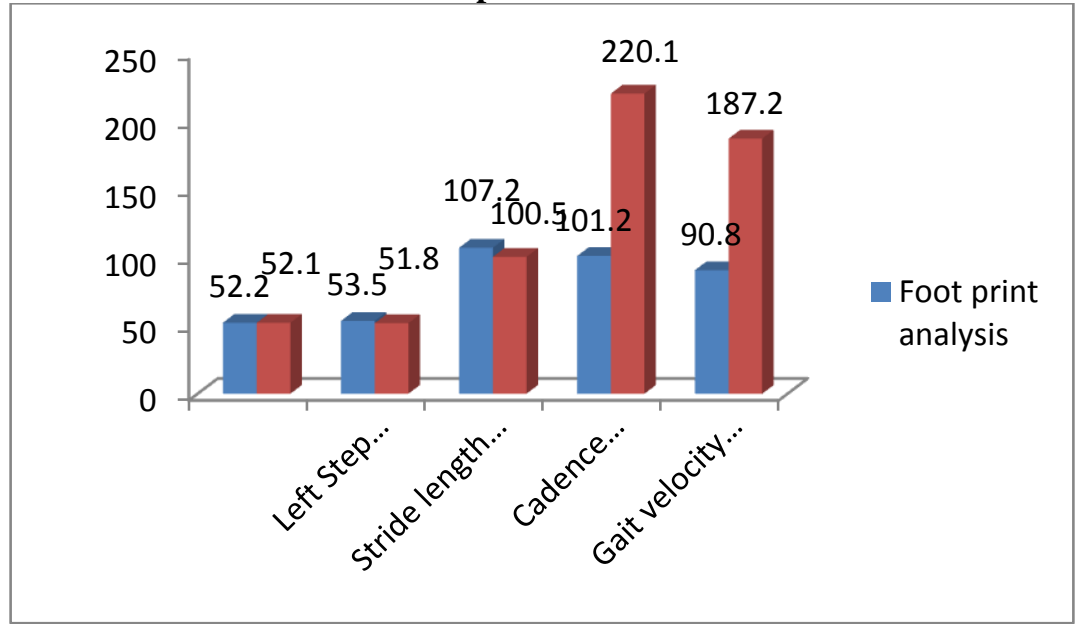

\section{Discussion:}

The study included 30 healthy subjects, which were made to walk a $10 \mathrm{~m}$ walkway after applying blue ink to their feet and immediately after that on the WIN TRACK. It was ensured that none of the patient was suffering from any kind of pain in the lower limbs or any systemic illness because it may alter the results. All the spatial gait parameters i.e. step length, stride length, cadence and gait velocity were calculated by using both the methods on the same day.

It was observed that there was a much difference between the data obtained by the two methods, which in turn was found with more variability in stride length, cadence and gait velocity. During the gait analysis it was found that while walking on the WIN TRACK when the patient came near the WIN TRACK the patient use to get conscious, which alters his/her gait parameters.. It was also found that it calculates the cadence of the person only based on the three steps taken on it and it gives very high values for the cadence accordingly. Also the surface of the WIN TRACK sheet is very soft. So while barefoot walking when the person came into contact with the sheet it alters his/ her all gait parameters.

Ramachandra et al(2012) stated that WIN TRACK platform 3 steps gait protocol shows better testretest reliability in terms of gait parameters. So it becomes very difficult for the participant to take 3 steps unconsciously on the WIN track and it further alters the result. If the participant is asked to take three steps only then the participant becomes conscious which further alters the gait parameters.

Shorten et al. 1989, Hughes et al. 1991, Rosenbaum et al. 1994, and Kernozek et al. 1996 proved that as the walking velocity increases the plantar pressure decrease at lateral foot, forefoot and mid toe, but it remains constant or increases at the sole of foot. This may be probably the reason that when participant walks with a particular velocity if plantar pressure is not detected by the sensors of the platform it will not record the readings of the particular parameters. As sometimes while taking the readings it was observed that participant has taken three steps but WIN TRACK platform was not able to record that.

\section{Conclusion}

The data obtained from the study revealed that a marked difference was seen in the gait parameters namely gait velocity, cadence and stride length when a comparison was made analyzing the gait parameters with a conventional method of footprints and WIN TRACK. Although there were no significant changes seen in the step length (right) and step length (left) statistically, but statistically insignificant differences were seen in these both parameters also. Future studies must be conducted on the patients with loss of sensation under the feet. Also studies must be conducted by embedding the WIN TRACK platform in the same surface of pathway and same color of pathway as well as platform. So as the participant should not get conscious during the protocol. Emphasis should also be made on the surface of the platform to make it as rigid as the walkway or vice versa such that it should be almost similar to the pathway.

\section{References:}

[1]. S. Ghoussayni, C. Stevens, S. Durham,D. wins. Assessment and validation of a simpleautomated method for the detection of gait events and intervals. Gait Posture 2004, 20, 266-272.

[2]. N.K. Boulgouris, D. Hatzinakos, K.N. Plataniotis. Gait recognition: A challenging signal processing technology for biometric identification. IEEE Signal Process. Mag. 2005, 22, 78-90.

[3]. K. Watanab, M. Hokari. Kinematical analysis and measurement of sports form. IEEE Trans. Syst. Man Cybern. Part A2006, 36, $549-557$. 
[4]. D.Y. Kwon, M. Gross. Combining body sensors and visual sensors for motion training. InProceedings of the 2005 ACM SIGCHI International Conference on Advances in Computer Entertainment Technology, Valencia, Spain, 15-17 June 2005 ; pp. 94-101.

[5]. L. Wang,W. Hu, T. Tan. Recent developments in human motion analysis. Pattern Recognit.2003, 36, 585-601.

[6]. Y. Wahab, N.A Bakar. Gait analysis measurement for sport application based on ultrasonicsystem. In Proceeding of the IEEE 15th International Symposium on Consumer Electronics, Singapore, 14-17 June 2011.

[7]. M.P.M. Steultjens, J. Dekker; M.E. van Baar. R.A.B. Oostendorp, J.W.J. Bijlsma. Range of joint motion and disability in patients with osteoarthritis of the knee or hip. Rheumatology 2000, 39, 955-961.

[8]. S. Kimmeskam, E.M. Hennig. Heel to toe motion characteristics in Parkinson patients during free walking. Clin. Biomech. 2001, $16,806-812$

[9]. M.H. Pope, T. Bevins, D.G Wilder, J.W. Frymoyer. The relationship between anthropometric, postural, muscular, and mobility characteristics of males ages 18-55. Spine 1985, 10, 644-648.

[10]. K. Turcot, R. Aissaoui, K. Boivin, M. Pelletier, N. Hagemeister, J.A de Guise. New accelerometric method to discriminate between asymptomatic subjects and patients with medial knee osteoarthritis during 3-D gait. IEEE Trans. Biomed. Eng. 2008, 55, 14151422 .

[11]. S.T. Moor, H.G. MacDougall,W.G. Ondo. Ambulatory monitoring of freezing of gait in Parkinson's disease. J. Neurosci. Methods 2008, 167, 340-348.

[12]. A. Salarian, H. Russmann, F.J.G. Vingerhoets, P.R. Burkhard, K. Aminian. Ambulatory monitoring of physical activities in patients with parkinsonapos's disease. IEEE Trans. Biomed. Eng. 2007, 54, 2296-2299.

[13]. 13. D.M. Gavrila, L.S. Davis. 3-D Model-based tracking of humans in action: A multi-view approach. In Proceedings of the IEEE Computer Vision and Pattern Recognition, San Francisco,CA, USA, 18-20 June 1996; pp. 73-79.

[14]. I.A. Karaulovaa, P.M. Hallb, A.D. Marshall. Tracking people in three dimensions using a hierarchical model of dynamics. Image Vis. Comput. 2002, 20, 691-700.

[15]. H. Furnée, Real-time motion capture systems. In Three-Dimensional Analysis of Human Locomotion; Allard, P., Cappozzo, A., Lundberg, A., Vaughan, C.L., Eds.; John Wiley \& Sons: Chichester, UK, 1997; pp. 85-108.

[16]. A. Cappozzo, U. Della Croce, A. Leardini, L. Chiari. Human movement analysis using stereophotogrammetry. Part 1: Theoretical background. Gait Posture 2005, 21, 186-196.

[17]. Chiari, L.; Della Croce, U.; Leardini, A.; Cappozzo, A. Human movement analysis using 1. Stereophotogrammetry. Part 2: Instrumental errors. Gait Posture 2005, 21, 197-211.

[18]. C.M. Kim, Eng, J.J. Magnitude and pattern of 3D kinematic and kinetic gait profiles in persons with stroke: Relationship to walking speed. Gait Posture 2004, 20, 140-146.

[19]. M. Casadio, P.G. Morasso, V. Sanguineti. Direct measurement of ankle stiffness during quiet standing: Implications for control modelling and clinical application. Gait Posture 2005, 21, 410-424.

[20]. P. Bonato. Wearable sensors/systems and their impact on biomedical engineering. Eng. Med.Biol. Mag. 2003, 22, 18-20.

[21]. M. Engin,A. Demirel, E.Z. Engin, M. Fedakar, M. Recent developments and trends in biomedical sensors. Measurement 2005, 37, $173-188$.

[22]. I.P.I. Pappas, M.R. Popovic, T. Keller, V. Dietz, M. Morari. A reliable gait phase detection system. IEEE Trans. Rehabil. Eng. 2001, 9, 113-125.

[23]. H.J. Luinge, P.H. Veltink. Inclination measurement of human movement using a 3-D accelerometer with autocalibration. IEEE Trans. Rehabil. Eng. 2004, 12, 112-121.

[24]. Observational Gait Analysis Handbook; the Professional Staff Association of Rancho Los Amigos Medical Center: Downey, CA, USA, 1989.

[25]. J. Parry. Gait Analysis Normal and Pathological Function; Slack Incorporated: Thorofare, NJ, USA, 1992.

[26]. W. Zijsltra, K. Aminian. Mobility assessment in older people: New possibilities and challenges. Eur. J. Aging 2007, 4, 3-12.

[27]. M.H. Westbrook. Accelerometers InAutomative Sensors; Westbrook, M.H., Turner, J.D., Eds.; Institute of Physics Publishers: Bristol, UK, 1994; pp. 150-174.

[28]. C.V.C Bouten, K.T.M. Koekkoek,M. Verduin, R. Kodde, J.D. Janssen. A triaxial accelerometer and portable data processing unit for the assessment of daily physical activity. IEEE Trans. Biomed. Eng. 1997, 44, 136-147. Zeng, H.; Zhao, Y. Sensing movement: Microsensors for body motion measurement. Sensors 2011, 11, 638-660.

[29]. B. Ayrulu-Erdem, B. Barshan, Leg motion classification with artificial neural networks using wavelet-based features of gyroscope signals. Sensors 2011, 11, 1721-1743.

[30]. P. Catalfamo,S. Ghoussayni, D. Ewins. Gait event detection on level ground and incline walking using a rate gyroscope. Sensors 2010, 10, 5683-5702.

[31]. O. Tuncel,K. Altun, B. Barshan. Classifying human leg motions with uniaxial piezoelectric gyroscopes. Sensors 2009, 9, 85088546.

[32]. D.L. Graham,H.A. Ferreira, P.P. Freitas. Magnetoresistive-based biosensors and biochips. TRENDS Biotechnol. 2004, 22, 455462.

[33]. R. Dai, R.B. Stein, B.J. Andrews, K.B. James, M. Wieler. Application of tilt sensors in functional electrical stimulation. IEEE Trans. Rehabil. Eng. 1996, 4, 63-72.

[34]. K.J. O’Donovan, R. Kamnik, D.T. O'Keeffe, G.M. Lyons. An inertial and magnetic sensor based technique for joint angle measurement. J. Biomech. 2007, 40, 2604-2611. Sensors 2012, 122275

[35]. J.H. Choi, J. Cho, J.H. Park, J.M. Eun, M.S. Kim. An efficient gait phase detection device based on magnetic sensor array. In Proceedings of the 4th Kuala Lumpur International Conference on Biomedical Engineering, Kuala Lumpur, Malaysia, 25-28 June 2008; Volume 21, pp. 778-781.

[36]. A.C. Nicol. A new flexible electrogoniometer with wide-spread applications. In International Series on Biomechanics X-B; Jonsson, B., Ed.; Human Kinetics Publishers Inc.: Champaign, IL, USA, 1987; pp. 1029-1033.

[37]. P.J. Rowe, A.C. Nicol, I.G. Kelly. Flexible goniometer computer system for the assessment of hip function. Clin. Biomech. 1989, 4, $68-72$.

[38]. K. Jaworek, M. Derlatka, M. Dominikowski, M. Investigation of human locomotion using penny \& giles electrogoniometer. In Proceedings of the SPIE_-The International Society for Optical Engineering, Jurata, Poland, 10 May 1998; Volume 3730, pp. 199_ 209.

[39]. P.J. Rowe,C.M. Myles,S.J. Hillmann, M.E. Hazlewood. Validation of flexible electrogoniometry as a measure of joint kinematics. Physiotherapy 2001, 87, 479-488.

[40]. T.O. Sato, G.A. Hansson, H.J.C.G. Coury. Goniometer crosstalk compensation for knee joint applications. Sensors 2010, 10, 999410005 . 
[41]. R. Roduit, P.A. Besse, J.P. Micallef. Flexible angular sensor. IEEE Trans. Instrum. Meas. 1998, 47, 1020-1022.

[42]. G.T. Laskoski, L.D.L Martins, S.F. Pichorim, P.J Abatti. Development of a telemetric goniometer. In Proceedings of the World Congress 2009-Medical Physics and Biomedical Engineering, Munich, Germany, 7-12 September 2009; Volume 25/IX, pp. $227-$ 230 .

[43]. M. Donno, E. Palange, F. Di Nicola, G. Bucci, F. Ciancetta. A flexible optical fiber goniometer for dynamic angular measurements. In Proceedings of the IEEE Instrument and Measurement Technology Conference, Warsaw, Poland, 1-3 May 2007; pp. 1-5.

[44]. M. Donno, E. Palange, F. Di Nicola, G. Bucci, F. Ciancetta. A new flexible optical fiber goniometer for dynamic angular measurements: application to human joint movement monitoring. IEEE Trans. Instrum. Meas. 2008, 57, 1614-1620.

[45]. F.H. Raab, E.B. Blood, T.C. Steiner, H.R. Jones. Magnetic position and orientation tracking system. IEEE Trans. Aerosp. Electron Syst. 1979, 15, 709-718.

[46]. A.D. Milne, D.G. Chess, J.A. Johnson, G.J.W. King. Accuracy of an electromagnetic tracking device: A study of the optimal operating range and metal interference. J. Biomech. 1996, 29, 791-793.

[47]. K. Kobayashi, L. Gransberg, E. Knutson, P. Nolen. A new system for three-dimensional gait recording using electromagnetic tracking. Gait Posture 1997, 6, 63-75.

[48]. P.M. Mills, S. Morrison, D.G. Lloyda, R.S. Barrett. Repeatability of 3D gait kinematics obtained from an electromagnetic tracking system during treadmill locomotion. J. Biomech. 2007, 40, 1504-1511. 